\title{
Pier Massimo Prosio, Stendhal e altri viaggiatori a Torino. Il viaggio letterario da Tasso a Nietzsche
}

\section{Annalisa Bottacin}

\section{(2) OpenEdition}

1 Journals

\section{Edizione digitale}

URL: http://journals.openedition.org/studifrancesi/9266

DOI: 10.4000/studifrancesi.9266

ISSN: 2421-5856

\section{Editore}

Rosenberg \& Sellier

\section{Edizione cartacea}

Data di pubblicazione: 1 juin 2008

Paginazione: 202-203

ISSN: 0039-2944

\section{Notizia bibliografica digitale}

Annalisa Bottacin, «Pier Massimo Prosio, Stendhal e altri viaggiatori a Torino. I/ viaggio letterario da Tasso a Nietzsche», Studi Francesi [Online], 154 (LII | I) | 2008, online dal 30 novembre 2015, consultato il 10 janvier 2021. URL: http://journals.openedition.org/studifrancesi/9266 ; DOI: https://doi.org/10.4000/ studifrancesi.9266

Questo documento è stato generato automaticamente il 10 janvier 2021.

\section{(c)}

Studi Francesi è distribuita con Licenza Creative Commons Attribuzione - Non commerciale - Non opere derivate 4.0 Internazionale. 


\title{
Pier Massimo Prosio, Stendhal e altri viaggiatori a Torino. Il viaggio letterario da Tasso a Nietzsche
}

\author{
Annalisa Bottacin
}

\section{NOTIZIA}

PIER MASSIMO PROSIO, Stendhal e altri viaggiatori a Torino. Il viaggio letterario da Tasso a

Nietzsche, Moncalieri, C.I.R.V.I., 2004 («Biblioteca del Viaggio in Italia. Studi»), pp. 260.

1 Il viaggio in Italia è continuamente contrassegnato da una riflessione intorno al luogo da visitare, in particolar modo la città, che si concatena in una serie di sequenze che conducono alla produzione di una storia. Partendo da tale investimento, atto a suscitare e produrre emozioni e ad intrecciare ricordi, Prosio nel suo bel volume dedicato a Stendhal (che è preceduto nella narrazione da una «Prefazione» (p.9) e da ritratti di letterati (pp.11-60) quali Tasso, Rousseau, Boswell e Sterne e ad altri nomi di grande prestigio, partiti alla conoscenza e alla scoperta del Bel Paese, ci conduce in quei percorsi torinesi dove l'artista si vede assegnare il ruolo di testimone di spazi nuovi, attraversati da sconfinamenti, che ben s'inseriscono nella sfera della produttività. Dei componenti della famiglia savoiarda dei de Maistre, non è al più noto Joseph (i cui scritti, fatto da segnalare, sono stati recentemente in parte riediti, a cura di Pierre Glandes per Laffond) che pone la sua attenzione Prosio, bensì al fratello minore Xavier de Maistre (pp. 61-74) «originale personalità di scrittore, creatore di brevi, affascinanti opere, ricche di spirito di osservazione e di humour» (p.63). Xavier, indomito combattente, decorato con la gran croce di San Maurizio e Lazzaro, fedelissimo alla corte sabauda, rivide Torino dopo aver combattuto nell'armata austro-russa tra i dragoni della Savoia, solo nel 1826, dopo una prima visita risalente al 1787. La città, da lui sempre considerata una seconda patria (i de Maistre sono savoiardi di Chambéry), è accuratamente descritta nel Voyage autour de ma chambre e nell'Expédition nocturne autour de ma chambre, da cui scaturisce veemente quella «partecipazione affettiva tutta 
particolare che si ha verso la propria terra» (p.65). Il primo soggiorno torinese di Stendhal (pp. 75-104), di soli due giorni, risale all'ottobre del 1801, con provenienza da $\mathrm{Bra}$, allorché, giovane tenente di cavalleria a titolo provvisorio, si reca in Italia al seguito dell'armata di riserva. In data 26 vendémiaire an X [18 octobre 1801] annota nel Journal: «Je vais à Turin avec le capitaine Frère et sa femme. J'y couche deux nuits. Je dîne deux fois à la citadelle chez le chef Contans... Je reviens le 28». Torino era comunque già nella sua memoria, l'aveva scoperta alla lettura dell'episodio di Mme Bazile nelle Confessions di Rousseau. «La Cittadella di Torino - nota Prosio - simbolo del potere militare in Piemonte e ormai occupata dalle truppe napoleoniche dunque, è il primo luogo 'stendhaliano' torinese in cui ci si imbatte» (pp. 78-79); la roccaforte è per altro già menzionata da Beyle in una nota del Journal del 4 ottobre. Seppur la città sabauda venga citata in un passaggio dell'Henry Brulard, risalente ad un presunto viaggio compiuto l'anno seguente, pare assai remota la possibilità di una visita a Torino di Beyle antecedente al novembre del 1813, con provenienza da Milano; un altro viaggio è presumibile nell'agosto del 1814, che comunque potrebbe condurre ad un 'tranello' stendhaliano, su cui il critico ben si cimenta. L'avvenimento si ricolloca infatti ancora una volta nell'universo del melomane Henri, innamorato dell'opera italiana, che tanto lo esaltava, ed in particolar modo relativamente ad una sua asserzione, sull'opera buffa, La Contessa di Colle Erboso di Pietro Generali, pseudonimo di Pietro Mercandetti (1773-1832), compositore biellese di successo fino all'affermazione di Rossini, del quale per certi aspetti le opere di Generali anticipano l'avvento. Stendhal scrive di averla ascoltata a Torino nell'agosto del 1814, ma Prosio asserisce che La Contessa fu portata sulle scene torinesi solo nel dicembre del 1831. Lo studioso si pone il quesito su quando e dove il grenoblese abbia ascoltato quella da lui definita una «musique charmante». Torino fu anche per Stendhal luogo d'instaurazione di importanti conoscenze e amicizie, tra cui quelle con l'avvocato Ferdinando Dal Pozzo, l'astronomo Giovanni Plana e il magistrato Carlo Guasco. Il ricordo di Torino in un altro letterato, Alphonse de Lamartine (pp. 105-128), che fu chargé d'affaires presso le ambasciate di Napoli e di Firenze, è quanto mai lusinghiero. In una lettera all'amico Aymon de Virieu, scrive: «Je ne me figurais une ville aussi belle que Turin» (p. 107), dopo il primo contatto con la città nel 1811, con la quale, per altro, ebbe forti legami, dovuti alle amicizie con alcuni giovani piemontesi, compagni di collegio a Belley dal 1803 al 1808, in particolare «Cesare Alfieri di Sostegno, figlio del marchese Carlo Emanuele, eminente personaggio che fu ministro del re di Sardegna a Parigi dal 1814 al 1828 e fratello di Costanza, futura sposa di Roberto d'Azeglio» (p. 108). Nel 1823, Lamartine corteggiò, dopo una gita alla Grande Chartreuse, Giulia Colbert marchesa di Barolo, un fatto che insieme ad avverse idee politiche, allontanerà l'autore delle Méditations dai nobili piemontesi. Anche JeanFrançois Champollion, (pp. 129-141) il noto orientalista, che confrontando nella celebre stele di Rosetta, scoperta durante la spedizione di Bonaparte in Egitto, il testo geroglifico e quello demotico con il testo greco, decifrò i geroglifici, fu ospite di Torino. In effetti vi si recò per visitare «lo straordinario complesso di antichità egizie che doveva dar origine al celebre museo» (p.131), giungendovi nel giugno del 1824 e prendendo alloggio all'Hôtel Feder, sito nell'attuale via Verdi. Grande fu l'entusiasmo di Champollion nella visita all'Accademia delle Scienze, atteso e ricevuto con onori dagli illustri accademici, e ancora maggior ammirazione provò al nuovo Museo Egizio, al punto di discriverlo, in idioma italiano, come "cosa stupenda» (p. 134). Haut lieu littéraire torinese, come rileva Prosio, era in Piazza Castello, l'Hôtel de l'Europe, dove tra tanti illustri viaggiatori sostò nell'estate del 1836 anche Honoré de Balzac (pp. 143-157), 
che subito ebbe a integrarsi con la società piemontese. Il critico fa accenno, tra l'altro, ad una gita a Rivalta Torinese, fatta dallo scrittore in compagnia di un misterioso «Marcel» (in realtà Mme Marbouty), del marchese Felice Carrone di San Tommaso e dell'abate Gazzera in un castello (vi è esposta una lapide a ricordo) «ove era ad attenderlo ed onorarlo la contessa Polissena di Benevello» (p.144), sposa del proprietario del maniero, il conte Cesare Della Chiesa di Benevello. Balzac «sollecitato dal luogo e dalle memorie» (p. 146) produrrà un breve racconto in francese antico, Le Chevalier de Saint-Martin, che sarà inserito nei Contes drolatiques. Furono giornate di grande interesse quelle trascorse a Torino dallo scrittore con Mme Marbouty, che per ovviare a pettegolezzi si travestì da uomo, facendosi passare per il suo segretario, appunto con il nome di «Marcel». Pur tuttavia l'inghippo venne presto alla luce; i torinesi si chiesero se sotto quelle vesti maschili si celasse George Sand, per di più anch'essa amica di Balzac, e l'argomento fu a lungo dibattuto, ma lo scrittore non confermò mai la presenza di Mme Marbouty; solo una volta, rientrato a Parigi, negò che la sua compagna fosse Gorge Sand.

Il pregevole volume che dedica i due ultimi capitoletti a Flaubert (pp.159-168) e al filosofo Nietzsche (pp. 169-184), aggiunge un'appendice che comprende due altri studi: «1958: la Torino di Lucius Burckhardt» (pp.185-193) e «Samuel Butler a Casale» (pp. 195-207). L'opera è sorretta da un corposo apparato di note (pp. 209-237) e da un indice dei nomi e dei luoghi (pp. 241-260). 